\title{
Clinical outcomes with use of radiation therapy and risk of transformation in early-stage follicular lymphoma
}

\author{
Fushen Sha $\mathbb{D}^{1}{ }^{1}$, Michelle Okwali ${ }^{1}$, Anna Alperovich ${ }^{1}$, Philip C. Caron ${ }^{1}$, Lorenzo Falchi $\mathbb{D}^{1}$, Audrey Hamilton ${ }^{1}$, Paul A. Hamlin ${ }^{1}$, \\ Steven M. Horwitz ${ }^{1}$, Erel Joffe (iD ${ }^{1}$, Niloufer Khan ${ }^{1}$, Anita Kumar ${ }^{1}$, Matthew J. Matasar ${ }^{1}$, Alison J. Moskowitz ${ }^{1}$, Ariela Noy (iD ${ }^{1}$, \\ Colette Owens ${ }^{1}$, Lia M. Palomba (iD) ${ }^{1}$, Ildefonso Rodriguez-Rivera ${ }^{1,2}$, David Straus ${ }^{1}$, Gottfried von Keudell ${ }^{1}$, Andrew D. Zelenetz ${ }^{1}$, \\ Joachim Yahalom ${ }^{3}$, Ahmet Dogan (iD) ${ }^{4}$, Heiko Schöder ${ }^{5}$, Venkatraman E. Seshan (iD) ${ }^{6}$, Gilles Salles (iD) ${ }^{1}$, Anas Younes (iD) ${ }^{1,7}$ and \\ Connie L. Batlevi (iD) ${ }^{1 凶}$
}

(c) The Author(s) 2022

Between 1998 and 2009, a total of 295 patients (median age 58, 53\% females) with newly diagnosed early-stage follicular lymphoma (FL) were managed at Memorial Sloan Kettering Cancer Center. Approximately half of patients (137, 46\%) underwent initial observation and half $(158,54 \%)$ immediate treatment: radiation alone $(n=108)$, systemic treatment alone $(n=29)$, or combined modality treatment $(n=21)$. Median follow-up was 8.4 years (range $0.3-17.2$ ), and 10 -year overall survival (OS) was $87.2 \%$. OS was similar between initiallyobserved and immediately-treated patients (hazard ratio [HR]: 1.25, 95\% Cl: $0.67-2.36, p=0.49$ ). For patients receiving radiation alone, 5-year OS was $98.0 \%$. Patients selected for systemic therapy alone had high-risk baseline features and had shorter OS than patients treated with radiation alone (HR 3.38, 95\% Cl 1.29-8.86, $p=0.01)$. Combined modality treatment did not yield superior survival compared with radiation alone $(P>0.05)$ but was associated with better progression-free survival $(\mathrm{HR} 0.36,95 \% \mathrm{Cl} 0.14-0.90, p=0.03)$. The rate of transformation increased steadily over time and was $4.2 \%$ at 5 years and $10.8 \%$ at 10 years. This modern-era analysis rationalized the role of initial observation in patients with early-stage FL although patients receiving radiation therapy also demonstrate excellent outcome.

Blood Cancer Journal (2022)12:29; https://doi.org/10.1038/s41408-022-00620-w

\section{INTRODUCTION}

Follicular lymphoma (FL) is the most common indolent lymphoma in the United States [1]. Initial management of FL depends on the stage at presentation. Approximately one-quarter of cases are early-stage (stage I-II) at diagnosis [1, 2]. Though patients with advanced-stage FL are considered incurable and are generally treated with chemoimmunotherapy when treatment is warranted, patients with stage I-II FL can be managed with a number of different approaches.

Initial observation has been reported to be an acceptable treatment option for patients with early-stage FL. However, this conclusion was largely based on small retrospective experiences before the use of modern imaging modalities $[3,4]$. The initial observation strategy has not been well validated in a larger patient population where 2-[ $\left[{ }^{18} \mathrm{~F}\right]$-Fluoro-2-deoxyglucose (FDG) positron emission tomography (PET) integrated with computed tomography (FDG-PET/CT) is routinely available. For patients receiving treatment after diagnosis, radiation therapy (RT) is an effective treatment option but relapse occurs in approximately $35 \%$ of cases [5-7]. Furthermore, disease progression primarily outside of the RT field can occur after RT [7, 8]. Studies investigating the benefit of combined modality treatment (CMT) drew distinct conclusions. The British National Lymphoma Investigation randomized trial found the addition of adjuvant oral chlorambucil to RT conferred no survival advantage, whereas the Trans-Tasman Radiation Oncology Group (TROG) found the addition of systemic chemotherapy regimen to RT was associated with longer response duration $[9,10]$. In a recent study with earlystage FL patients staged with PET, systemic treatment without rituximab maintenance failed to improve progression-free survival (PFS) [5]. The evidence guiding clinical management of early-stage FL patients in the era of modern imaging modality has been sparse. After completing therapy, patients with FL undergo regular monitoring to capture disease progression and histological transformation. However, the pattern of histological transformation has not been well illustrated in early-stage FL to inform longterm surveillance strategy.

To address these knowledge gaps, we initiated this single-center, retrospective analysis of patients with early-stage $\mathrm{FL}$, including a subpopulation who were staged with PET and bone marrow examination. We aimed to determine survival patterns and risk of transformation in these patients, as well as comparing the clinical

\footnotetext{
${ }^{1}$ Department of Medicine, Lymphoma Service, Memorial Sloan Kettering Cancer Center, New York, NY, USA. ${ }^{2}$ Currently employed at NEXT Oncology / Texas Oncology, San Antonio, TX, USA. ${ }^{3}$ Department of Radiation Oncology, Memorial Sloan Kettering Cancer Center, New York, NY, USA. ${ }^{4}$ Department of Pathology, Hematopathology Service,

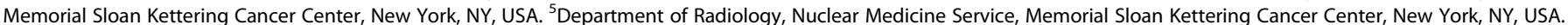
${ }^{6}$ Department of Epidemiology and Biostatistics, Memorial Sloan Kettering Cancer Center, New York, NY, USA. ${ }^{7}$ Currently employed at AstraZeneca, Wilmington, DE, USA. email: leec@mskcc.org
} 


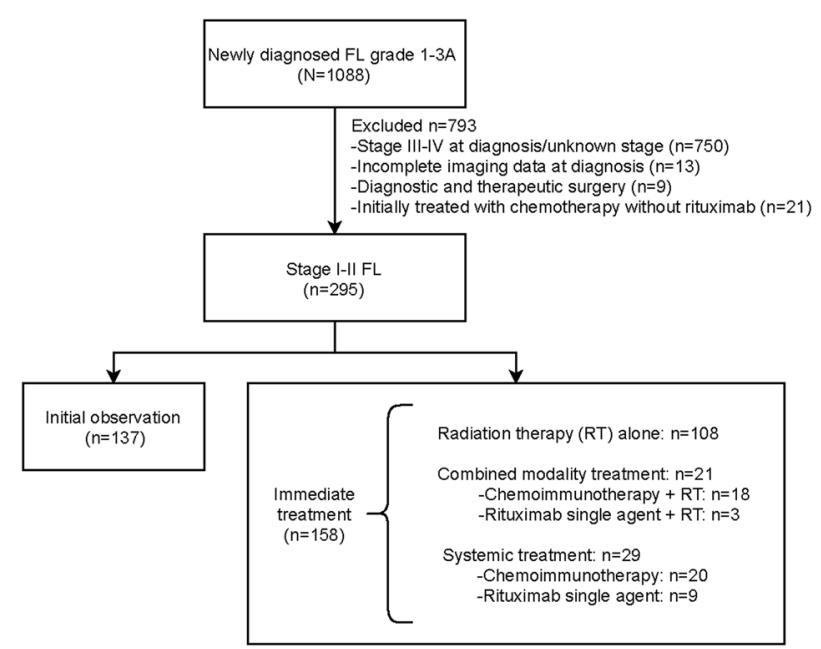

Fig. 1 CONSORT diagram of eligible patients with follicular lymphoma. Patients with grade 1-3 A, stage I-Il follicular lymphoma (FL) diagnosed between 1998 and 2009 and managed at MSK were identified. FL follicular lymphoma, RT radiation therapy.

outcomes (a) for initial observation $>6$ months vs immediate treatment, and (b) for immediate treatment with systemic therapy vs radiation therapy vs combined modality therapy.

\section{METHODS}

The study was approved by the institutional review board of Memorial Sloan Kettering Cancer Center (MSK). We identified 1088 patients with grade 1-3 A FL who were managed at MSK between 1998 and 2009. Study eligibility criteria included age $\geq 18$ years, stage I-II disease, MSK confirmed FL diagnosis, and no prior treatment for FL. We excluded 793 patients from this analysis: 750 had stage III-IV or an unknown stage of disease at diagnosis, 13 had incomplete imaging data at diagnosis, 9 patients had diagnostic and therapeutic surgery, and 21 patients had been treated with chemotherapy without rituximab, which does not represent the current standard of care (Fig. 1). Thus, 295 patients with histologically confirmed FL who were managed and followed at MSK were included in this analysis.

The subpopulation of patients who were completely staged $(n=154)$ had stage I-II disease confirmed with both PET scan and bone marrow examination. The other 141 patients were provisionally staged as stage I-II, i.e., staged with computed tomography without an FDG-PET or a bone marrow biopsy. All pathology was reviewed by MSK pathologists at the time of diagnosis and treatment.

For our analysis, we classified patients based on the length of time between diagnosis and start of treatment. Patients whom we considered to have immediate treatment were started on localized or systemic treatment within 6 months of diagnosis. Conversely, patients initially observed were observed for longer than 6 months.

Overall survival (OS) is measured from the date of diagnosis until death from any causes. PFS is defined as lasting from therapy initiation until disease progression or death from any cause. Multiple imputation was used to impute the Follicular Lymphoma International Prognostic Index (FLIPI) for missing data. We used Cox regression to estimate hazard ratios (HR) and $95 \%$ confidence intervals (Cl) for associations of treatment modality with OS and PFS, adjusting for imputed FLIPI.

To understand the pattern of disease-specific cause of death and histological transformation of FL following diagnosis, we used a competing risks analysis. For disease-specific cause of death, patients can experience death from lymphoma progression or death from unknown causes. Patient deceased from lymphoma-unrelated causes of death were censored. For histological transformation, a patient can experience one of two mutually exclusive events-pathologically confirmed transformed FL (tFL) or death without tFL. The time origin was set at the time of diagnosis. The causespecific event rates were plotted against time after diagnosis [11].

Statistical significance was defined as $P<0.05$, and all statistical analyses were completed with R 3.5.0. Demographics were compared between patients using t-test, Fisher's exact, and ANOVA test, as appropriate.

\section{RESULTS}

\section{Patient characteristics}

We identified 295 eligible patients diagnosed with grade 1-3 A, stage I-II FL managed at Memorial Sloan Kettering Cancer Center from 1998 to 2009 (Fig. 1). Baseline characteristics at diagnosis are summarized in Table 1. Among 295 patients, 154 patients were staged completely with PET and bone marrow examination. The median follow-up of all 295 eligible patients was 8.4 years (range $0.3-17.2$ ), and median age of all patients was 58 years (interquartile range [IQR] 48-67). More than half of patients were diagnosed with stage I disease $(n=172 ; 58 \%)$ and $42 \%(n=123)$ had stage II disease. The Follicular Lymphoma International Prognostic Index (FLIPI) risk score at diagnosis was available for $81 \%$ of patients (239/295), with low-risk and intermediate/highrisk disease representing $87 \%$ and $13 \%$, respectively. Baseline demographics were not significantly different between all eligible patients and patients completely staged with PET and bone marrow examination, except that the completely staged subpopulation was less likely to be initially observed: $36 \%$ of completely staged patients vs. $46 \%$ of all patients $(p=0.03)$ (Table 1, Supplemental Fig. S1).

Baseline features between patients who were initially observed and immediately treated were also compared (Supplemental Table S1). Patients immediately treated were more likely to have stage I disease than initially-observed patients $(71 \%$ vs. $44 \%, P<$ $0.001)$, as well as grade $3 \mathrm{~A}$ pathology $(20 \%$ vs. $7 \%, P<0.01)$ and confirmed bone marrow negativity at diagnosis $(84 \%$ vs. $64 \%, P<$ 0.001). Other features were not statistically different between the two groups. Interestingly, age at diagnosis was not a major determinant in selecting patients for observation or immediate treatment (Supplemental Fig. S2).

\section{Baseline demographics by management approaches}

Baseline features stratified by the management approaches are shown in Table 2 and Supplemental Table S1. Management approaches included initial observation $(n=137)$ and immediate treatment $(n=158)$. For immediately treated patients, 21 received combined modality treatment (CMT), 108 had radiation therapy alone (RT), and 29 received systemic therapy alone. Sites of radiation therapy are further described in Supplemental Fig. S3. Patients treated with CMT all received radiation therapy, plus either single-agent rituximab $(n=3)$ or rituximab in combination with cyclophosphamide, doxorubicin, vincristine, and prednisone (R-CHOP, $n=18$ ). Of the patients who received systemic therapy alone, nine patients received single-agent rituximab, and 20 patients had chemoimmunotherapy. Of the total 158 patients who were immediately treated, only $3 \%(5 / 158)$ patients received rituximab maintenance. Patients treated with RT and CMT had similar baseline characteristics except that more patients treated with CMT had grade 3 A pathology (62\% vs. $12 \%, P<0.001$, Table 2). Compared with patients treated with radiation therapy alone, patients selected for a systemic regimen alone were more likely to have high-risk baseline features, including stage II disease $(86 \%$ vs. $15 \%, p<0.001)$, intermediate-high risk FLIPI score (26\% vs. $7 \%$, $p=0.06)$, elevated LDH ( $24 \%$ vs. $6 \%, p=0.09)$, involvement of more than 4 nodal areas ( $7 \%$ vs. $0 \%, p=0.05)$, bulky disease (defined as lesion $>7 \mathrm{~cm}, 41 \%$ vs. $6 \%, p<0.001$ ), and high SUVmax (defined as SUVmax $>12,32 \%$ vs. $2 \%, p=0.01$ ) (Table 2).

\section{Survival after diagnosis}

The median OS for all comers was not reached based on 39 observed deaths. The 5-, 10-, and 15-year OS rates for all patients were $95.0 \%$ (95\% confidence interval [Cl] 0.925-0.976), 87.2\% (95\% Cl 0.829-0.918), and 71.5\% (95\% Cl 0.595-0.860), respectively (Fig. 2A).

Patients who were completely staged with PET and bone marrow examination had significantly superior OS compared with patients staged provisionally, with median OS being not reached 
Table 1. Baseline characteristics of patients with grade 1-3 A, stage I-II follicular lymphoma managed at MSK between 1998 and 2009.

\begin{tabular}{|c|c|c|c|c|c|}
\hline \multirow[b]{3}{*}{ Characteristic } & \multirow{2}{*}{\multicolumn{2}{|c|}{$\begin{array}{l}\text { All patients } \\
(N=295)\end{array}$}} & \multirow{2}{*}{\multicolumn{2}{|c|}{$\begin{array}{l}\begin{array}{l}\text { Completely } \\
\text { staged }^{\mathrm{a}}\end{array} \\
(N=154)\end{array}$}} & \multirow[b]{3}{*}{$\boldsymbol{P}$} \\
\hline & & & & & \\
\hline & No. & $\%$ & No. & $\%$ & \\
\hline Age: Median (IQR) & $58(48-67)$ & $57(47-65)$ & 0.25 & & \\
\hline \multicolumn{6}{|l|}{ Sex } \\
\hline Female & 156 & 53 & 78 & 51 & 0.69 \\
\hline Male & 139 & 47 & 76 & 49 & \\
\hline \multicolumn{6}{|l|}{ Stage } \\
\hline 1 & 172 & 58 & 94 & 61 & 0.61 \\
\hline ॥ & 123 & 42 & 60 & 39 & \\
\hline \multicolumn{6}{|l|}{ Grade } \\
\hline $1-2$ & 227 & 86 & 121 & 83 & 0.39 \\
\hline $3 \mathrm{~A}$ & 36 & 14 & 25 & 17 & \\
\hline Unknown & 32 & 8 & & & \\
\hline \multicolumn{6}{|l|}{ FLIPI } \\
\hline Low & 209 & 87 & 117 & 89 & 0.74 \\
\hline Intermediate-High & 30 & 13 & 14 & 11 & \\
\hline Unknown & 56 & 23 & & & \\
\hline \multicolumn{6}{|l|}{ LDH } \\
\hline Elevated & 25 & 12 & 13 & 11 & 0.86 \\
\hline Normal & 189 & 88 & 108 & 89 & \\
\hline Unknown & 81 & 33 & & & \\
\hline \multicolumn{6}{|l|}{ Hemoglobin } \\
\hline Decreased & 15 & 6 & 7 & 5 & 1.00 \\
\hline Normal & 244 & 94 & 130 & 95 & \\
\hline Unknown & 36 & 17 & & & \\
\hline \multicolumn{6}{|l|}{ Nodal areas } \\
\hline$>4$ & 8 & 3 & 3 & 2 & 0.76 \\
\hline$\leq 4$ & 287 & 97 & 151 & 98 & \\
\hline \multicolumn{6}{|l|}{ Bulky disease $(>7 \mathrm{~cm})$} \\
\hline Yes & 30 & 16 & 14 & 12 & 0.50 \\
\hline No & 160 & 84 & 100 & 88 & \\
\hline Unknown & 105 & 40 & & & \\
\hline \multicolumn{6}{|l|}{ PET staged } \\
\hline Yes & 206 & 70 & 154 & 100 & NA \\
\hline No & 89 & 30 & 0 & 0 & \\
\hline \multicolumn{6}{|l|}{ SUVmax } \\
\hline$>12$ & 13 & 10 & 9 & 9 & 1.00 \\
\hline$\leq 12$ & 123 & 90 & 90 & 91 & \\
\hline Unknown & 159 & 55 & & & \\
\hline \multicolumn{6}{|l|}{ Bone marrow negativity } \\
\hline Yes & 220 & 75 & 154 & 100 & NA \\
\hline Unknown & 75 & 25 & 0 & 0 & \\
\hline \multicolumn{6}{|l|}{ Initial management } \\
\hline Initial observation & 137 & 46 & 55 & 36 & 0.03 \\
\hline Immediate treatment & 158 & 54 & 99 & 64 & \\
\hline
\end{tabular}

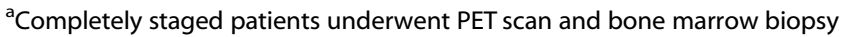
at diagnosis.

IQR interquartile range, FLIPI Follicular Lymphoma International Prognostic Index, LDH lactate dehydrogenase, PET positron emission tomography, SUV standard uptake value.

in either group (hazard ratio [HR] 0.45, 95\% Cl 0.22-0.92, log-rank $p=0.02$ ). For completely staged patients, the 5 - and 10-year OS rates were $96.7 \%(95 \% \mathrm{Cl} 0.939-0.996)$ and $92.1 \%(95 \%$ $\mathrm{Cl} 0.873-0.972)$, respectively. For provisionally staged patients, the
5- and 10-year OS rates were $93.2 \%(95 \% \mathrm{Cl} 0.890-0.976)$ and $82.6 \%$ (95\% Cl 0.757-0.901), respectively (Fig. 2B).

FLIPI score at diagnosis was prognostic for OS, with patients in the low-risk category demonstrating better survival than patients in the intermediate/high-risk category (HR 0.22, 95\% Cl 0.10-0.47, log-rank $P<0.001)$. Ten-year OS rates for patients with low and intermediate-high risk FLIPI were $91.4 \%$ and $71.1 \%$, respectively (Fig. 2C).

\section{Impact of initial observation on survival}

Fifty-four percent of all patients $(158 / 295)$ required immediate treatment after diagnosis, and 46\% (137/295) underwent initial observation. Patients completely staged were more likely to be immediately treated after diagnosis, with only $36 \%$ of completely staged patients (55/154) managed with initial observation (Table 1). However, the higher rate of initial observation in the provisionallystaged patients did not adversely affect OS for all patients (HR $1.25,95 \% \mathrm{Cl} 0.67-2.36$, log-rank $p=0.49$ ), nor for the completelystaged subpopulation (HR 1.07, 95\% Cl 0.31-3.65, log-rank $p=$ 0.92) (Fig. 3A, B). The median duration of observation in the initially-observed group $(n=137)$ was 5.62 years $(95 \% \mathrm{Cl}$ 3.95-10.41, Fig. 3C).

For all initially-observed stage I patients, OS was similar to that of immediately treated patients (HR 1.51, 95\% Cl 0.56-4.07, logrank $p=0.41$, Fig. 3D). For stage I patients who were completely staged, OS between initially-observed and immediately-treated patients was not significantly different $(\mathrm{HR} \quad 4.01,95 \% \mathrm{Cl}$ $0.66-24.29$, log-rank $p=0.13$, Fig. 3E). The median duration of observation for initially-observed stage I patients was 4.73 years (95\% Cl 2.66-not reached [NR], Fig. 3F). For stage II patients, those who were initially observed also had comparable OS to those who were immediately treated (Supplemental Fig. S4A, B). The median duration of observation for initially-observed stage II patients was 6.90 years (95\% Cl 4.15-NR, Supplemental Fig. S4C).

For patients who were initially observed and eventually treated (78/137), the median time to first treatment was 2.52 years (range $0.50-13.33$ years). For patients who were immediately treated after diagnosis, the median time from diagnosis to treatment was 2.2 months ( 0.18 years, range $0.00-0.48$ years).

\section{Survival by management approach}

Unadjusted Kaplan-Meier curves illustrate a difference in OS and PFS (Fig. 4). After adjustment for FLIPI, patients treated with CMT had similar OS compared with patients treated with radiation therapy (HR not available due to sparsity of events, $p=1.00$, Table 3 ), but had longer PFS (HR: 0.36, 95\%: 0.14-0.90, $p=0.03$, Table 3). Patients selected for systemic treatment had shorter OS vs patients treated with radiation therapy (HR: 3.38, 95\%Cl: 1.29-8.86, $p=0.01$, Table 3) despite statistically similar PFS (HR: $1.53,95 \% \mathrm{Cl}: 0.88-2.65, p=0.13$, Table 3 ). Given the prolonged observation time for patients who underwent initial observation in our study, PFS after treatment commencement was not analyzed for patients who were initially observed to avoid lead-time bias and stage migration after observation. Nevertheless, OS since diagnosis after initial observation was not significant abbreviated when compared with patient who were treated with radiation therapy (HR: $1.30,95 \% \mathrm{Cl}: 0.60-2.81, p=0.51$, Table 3). Observed trends of survival differences were maintained in subgroup of patients who were completely staged (Table 4).

Completely staged patients treated with CMT had an excellent 5 -year OS rate of $100 \%$. In comparison, the 5-year OS rates after management with either observation, RT, or systemic treatment in completely staged patients were $98.1 \%$, 98.4\%, and $84.2 \%$, respectively.

Cumulative incidence curves demonstrating disease-specific cause of death were analyzed by competing risk analysis, where patients experienced either death from lymphoma progression or death from unknown causes. Patients treated with systemic 
Table 2. Baseline characteristics based on management approach.

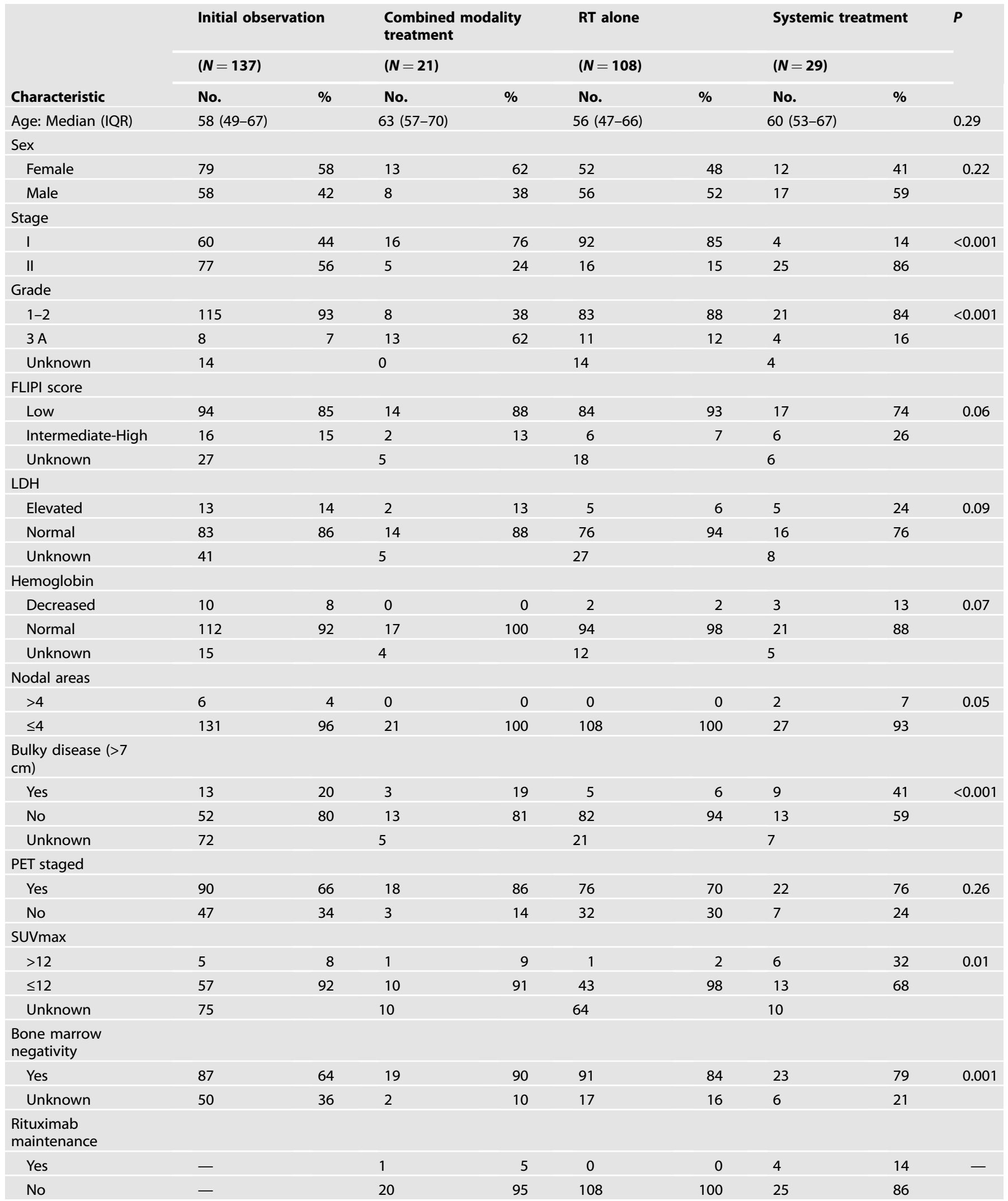

$R T$ radiation therapy, OBS observation, CMT combined modality treatment, IQR interquartile range, FLIPI Follicular Lymphoma International Prognostic Index, $L D H$ lactate dehydrogenase, PET positron emission tomography, SUV standard uptake value. 
A All patients, stage $\mathrm{I}-\mathrm{II}(\mathrm{n}=295)$

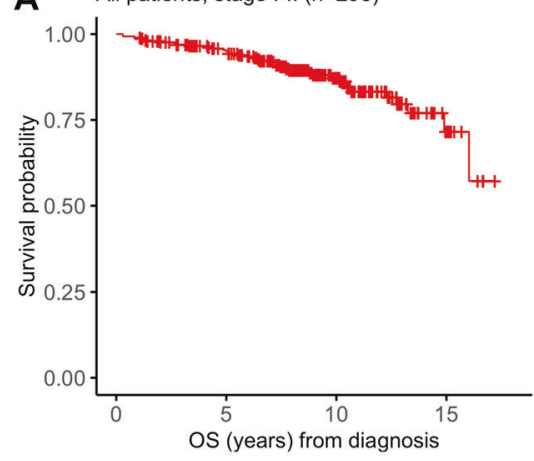

Number at risk 295 249 $98 \quad 12$

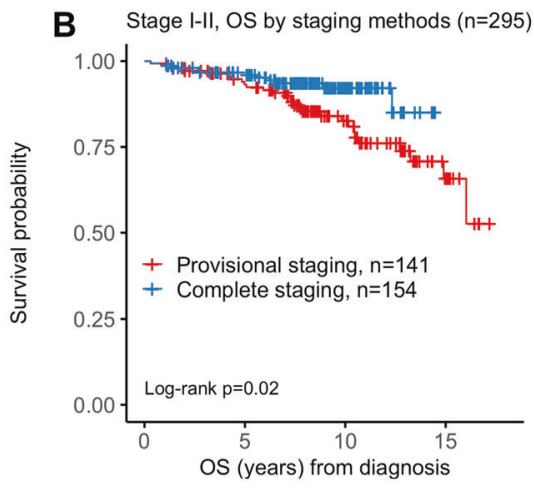

Number at risk Provisional 141
OS (years) from diagnosis

$\begin{array}{lll}117 & 55 & 12 \\ 132 & 43 & 0\end{array}$

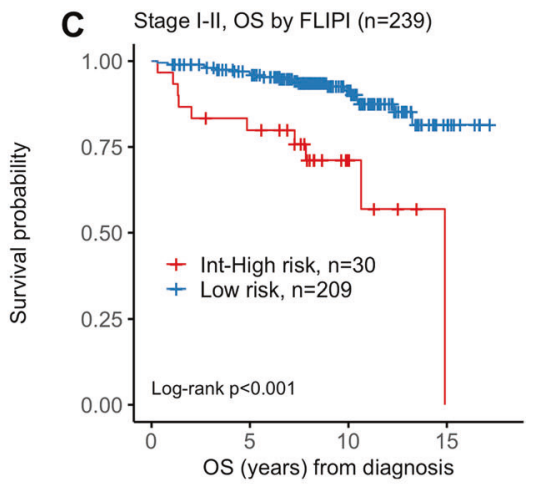

Number at risk

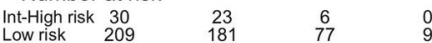

Fig. 2 Kaplan-Meier plots of survival after diagnosis. A Overall survival (OS) for all patients with stage I-II FL ( $n=295)$. B OS stratified by staging methods $(n=295)$. C OS stratified by the FLIPI risk category at diagnosis $(n=239)$.

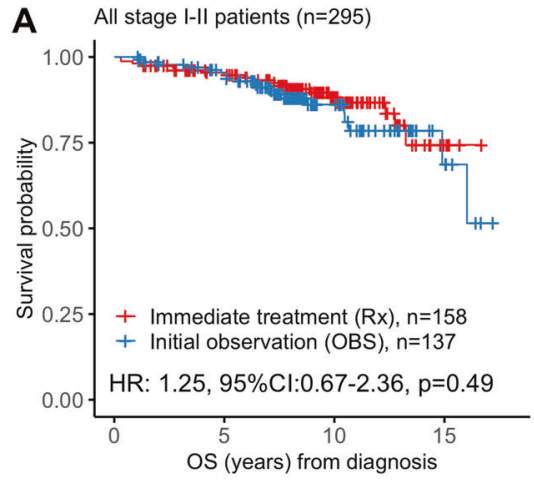

Number at risk $\begin{array}{ll}\text { Rx } & 158 \\ \text { OBS } & 137\end{array}$

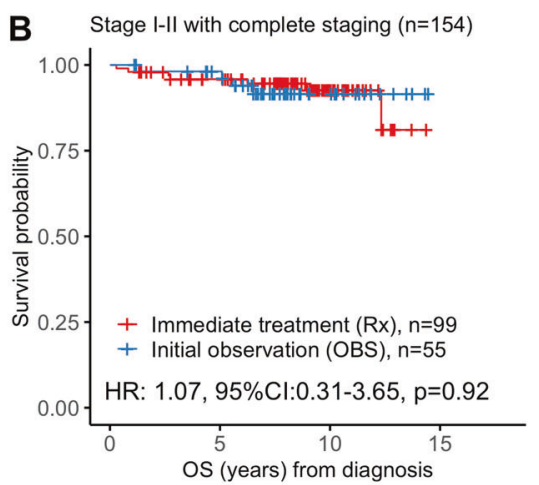

Number at risk $\begin{array}{lr}\text { Rx } & 99 \\ \text { OBS } & 55\end{array}$
C Stage I-II patients initially observed $(n=137)$

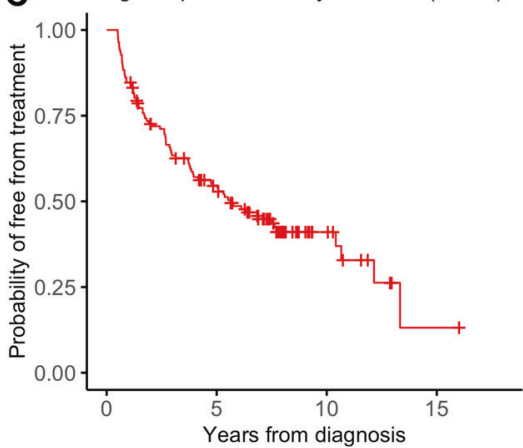

Number at risk
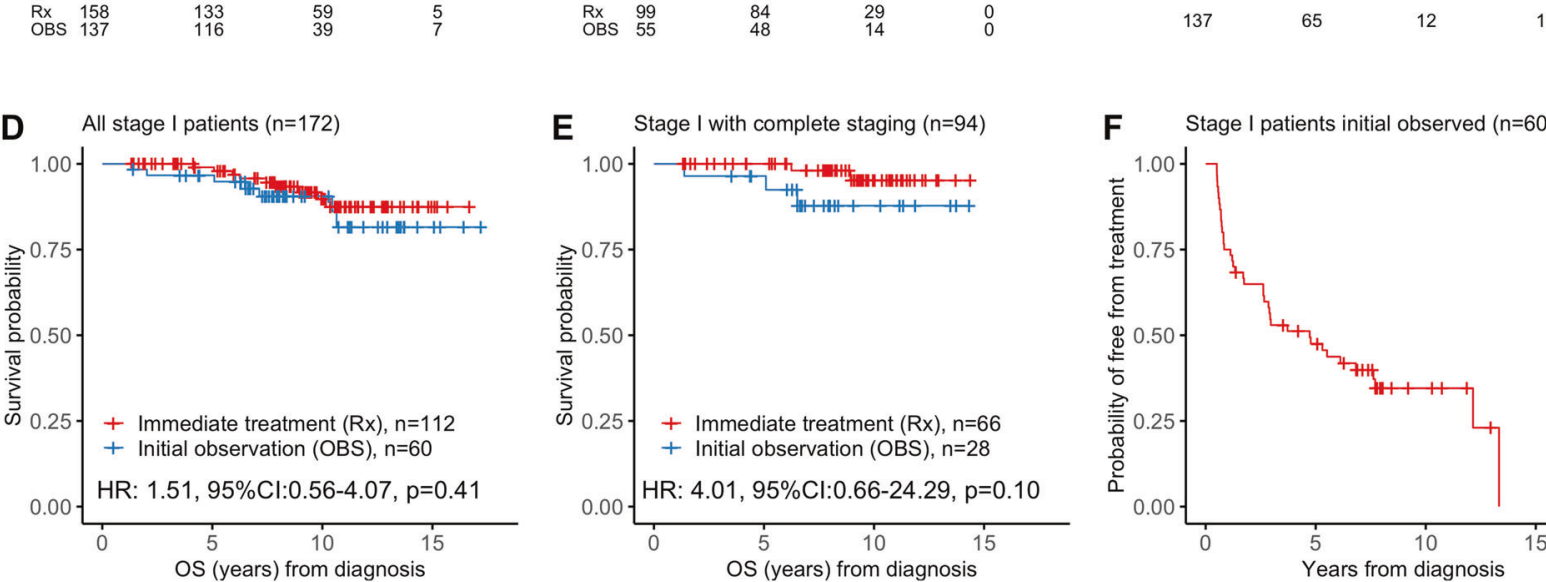

Number at risk

$\begin{array}{ll}\text { Rx } & 112 \\ \text { OBS } & 60\end{array}$

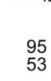

\section{E}

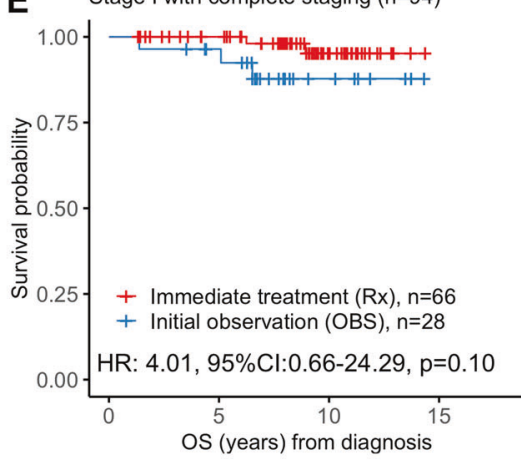

$$
\text { Number at risk }
$$

$\begin{array}{ll}\text { RX } & 66 \\ \text { OBS } & 28\end{array}$

$$
\begin{aligned}
& 56 \\
& 24
\end{aligned}
$$

$$
\begin{array}{ll}
20 & 0 \\
7 & 0
\end{array}
$$

\section{48}

29
14

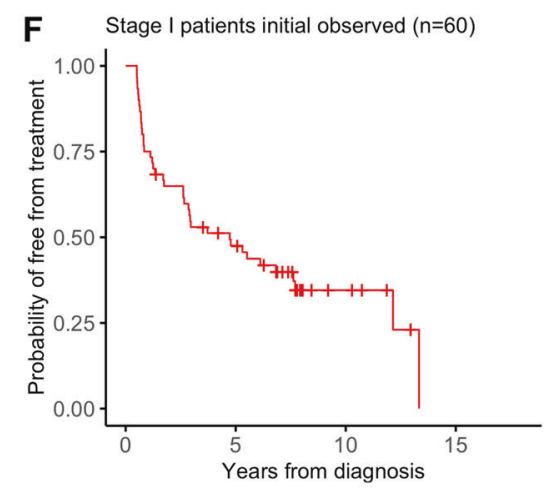

Number at risk

$$
60
$$

Fig. 3 Kaplan-Meier plots of survival and duration of observation after diagnosis. A Overall survival (OS) stratified by initial observation versus immediate treatment in all patients with stage I-II FL $(n=295)$. B OS stratified by initial observation versus immediate treatment in patients with stage I-II FL who were completely staged with PET scan and bone marrow biopsy at diagnosis ( $n=154$ ). C Duration of observation in all patients with stage I-II FL managed with initial observation $(n=137)$. D OS stratified by initial observation versus immediate treatment in all patients with stage I FL $(n=172)$. E OS stratified by initial observation versus immediate treatment in patients with stage I FL who were completely staged with PET scan and bone marrow biopsy at diagnosis $(n=94)$. F Duration of observation in all patients with stage I FL managed with initial observation $(n=60)$.

therapy had higher risk of death due to lymphoma progression after adjustment for FLIPI ( $p=0.001$, Supplemental Fig. S5A) whereas the risk of death from unknown causes was similar to the rest of groups after FLIPI adjustment ( $p=0.73$, Supplemental Fig. $\mathrm{S} 5 \mathrm{~A})$. The pattern was confirmed in subgroup of patients with complete staging (Supplemental Fig. S5B).

\section{Transformation}

We investigated patterns of transformation in early-stage FL. The patterns of biopsy-proven histological transformation and death without transformation are shown in Fig. 5. The rate of transformation, and the rate of death without transformation, steadily increase with increasing time after diagnosis. The risk of transformation at 5 


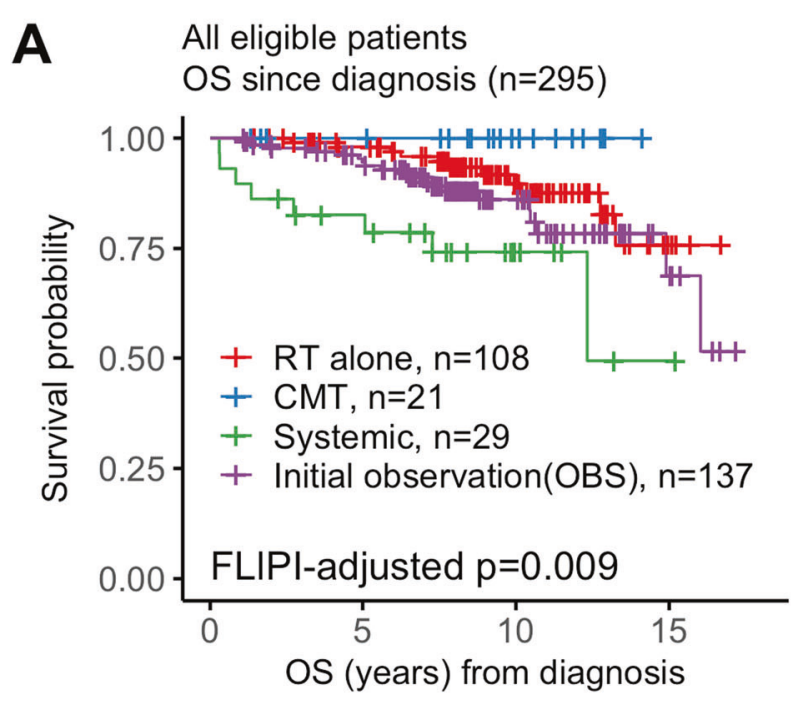

Number at risk

$\begin{array}{lcccc}\text { RT alone } & 108 & 94 & 43 & 4 \\ \text { CMT } & 21 & 18 & 9 & 0 \\ \text { Systemic } & 29 & 21 & 7 & 1 \\ \text { Initial OBS } & 137 & 116 & 39 & 7\end{array}$

C

Completely staged patients

OS since diagnosis $(n=154)$

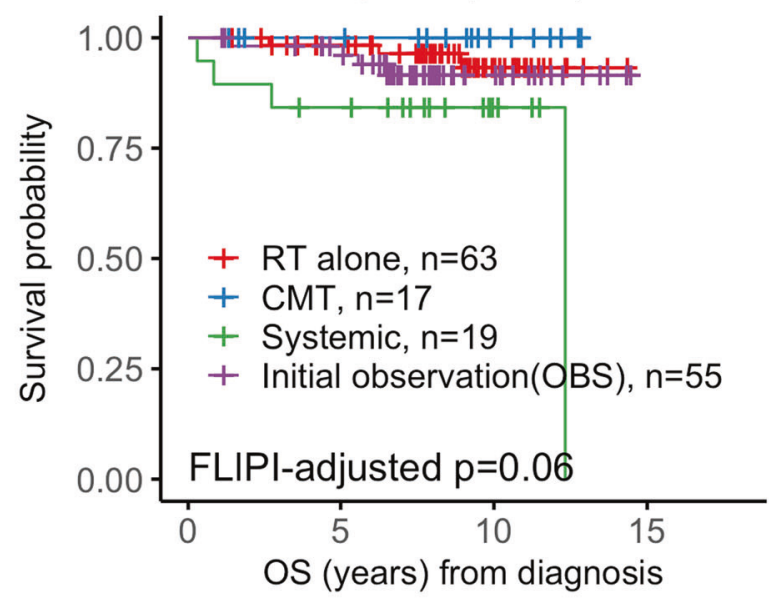

Number at risk

$\begin{array}{llccc}\text { RT alone } & 63 & 55 & 18 & 0 \\ \text { CMT } & 17 & 14 & 6 & 0 \\ \text { Systemic } & 19 & 15 & 5 & 0 \\ \text { Initial OBS } & 55 & 48 & 14 & 0\end{array}$

B All eligible patients

PFS since treatment $(n=158)$

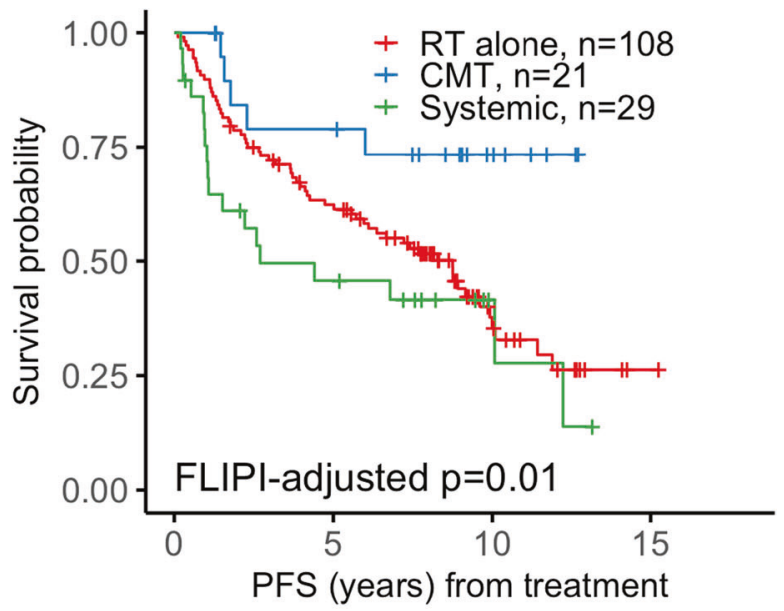

Number at risk

$\begin{array}{lcccc}\text { RT alone } & 108 & 63 & 15 & 1 \\ \text { CMT } & 21 & 15 & 6 & 0 \\ \text { Systemic } & 29 & 12 & 3 & 0\end{array}$

D Completely staged patients PFS since treatment $(n=99)$

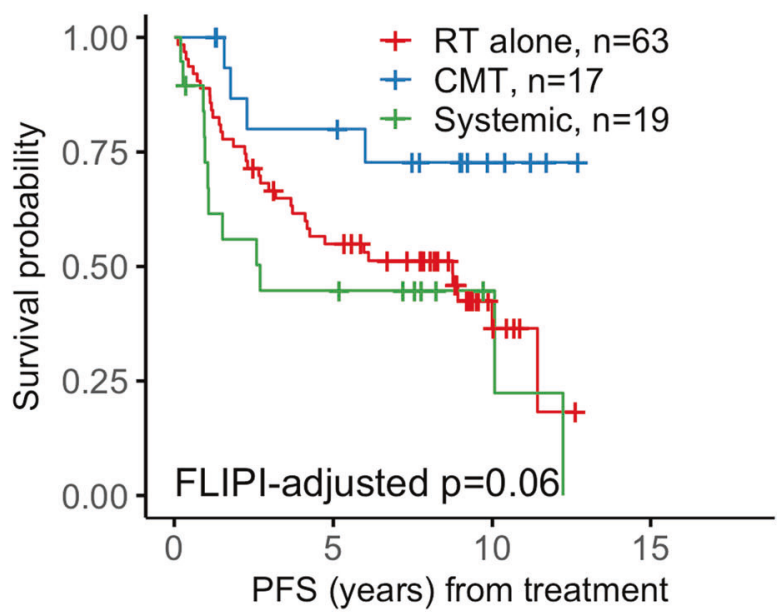

Number at risk

$\begin{array}{lcccc}\text { RT alone } & 63 & 33 & 6 & 0 \\ \text { CMT } & 17 & 12 & 4 & 0 \\ \text { Systemic } & 19 & 8 & 2 & 0\end{array}$

Fig. 4 Unadjusted Kaplan-Meier curves with $p$-values adjusted for FLIPI. A, B Survival analysis to compare outcomes by type of treatment received in all eligible stage I-II patients. A overall survival (OS) after diagnosis, B progression-free survival (PFS) after treatment, and C, D Survival analysis to compare outcomes by type of treatment received in stage I-II FL patients who were completely staged. C OS after diagnosis. D PFS after treatment.

and 10 years after diagnosis was $4.2 \%$ and $10.8 \%$, respectively. The risk of death without transformation at 5 and 10 years after diagnosis was $3.9 \%$ and $10.8 \%$, respectively. The aforementioned competing risks were not significantly different in patient who were initially observed versus in patient who were immediately treated (Supplemental Fig. S6). Patients selected for systemic treatment alone was observed to have higher risk of transformation at 5 years (18.4\%) and 10 years (18.4\%, figure not shown). 
Table 3. Survival analysis in all eligible patients with stage I-Il follicular lymphoma based on management approach.

\begin{tabular}{|c|c|c|c|c|c|}
\hline & & $\begin{array}{l}\text { Initial observation } \\
(N=137)\end{array}$ & $\begin{array}{l}\text { Combined modality } \\
\text { treatment } \\
(N=21)\end{array}$ & $\begin{array}{l}\text { Radiation therapy alone } \\
(N=108)\end{array}$ & $\begin{array}{l}\text { Systemic treatment } \\
(N=29)\end{array}$ \\
\hline $\begin{array}{l}\text { OS since } \\
\text { diagnosis }\end{array}$ & 5-year (95\% Cl) & $94.6 \%(0.907-0.986)$ & $100.0 \%(1.000-1.000)$ & $98.0 \%(0.953-1.000)$ & $82.6 \%(0.699-0.977)$ \\
\hline \multirow{2}{*}{$\begin{array}{l}\text { PFS since } \\
\text { treatment }\end{array}$} & 5-year $(95 \% \mathrm{Cl})$ & \multirow[t]{2}{*}{-} & $78.9 \%(0.626-0.996)$ & $62.3 \%(0.537-0.723)$ & $45.7 \%(0.304-0.689)$ \\
\hline & $\mathrm{HR}^{\mathrm{a}}(95 \% \mathrm{Cl})$ & & $0.36(0.14-0.90), p=0.03$ & Reference & $\begin{array}{l}1.53(0.88-2.65) \\
p=0.13\end{array}$ \\
\hline
\end{tabular}

${ }^{a}$ Hazard ratio and corresponding $p$-value was adjusted for the Follicular Lymphoma International Prognostic Index (FLIPI). OS overall survival, HR hazard ratio, Inf infinity, PFS progression-free survival.

Table 4. Survival analysis in patients with stage I-II follicular lymphoma confirmed with positron emission tomography (PET) and bone marrow examination.

\begin{tabular}{|c|c|c|c|c|c|}
\hline & & $\begin{array}{l}\text { Initial observation } \\
(N=55)\end{array}$ & $\begin{array}{l}\text { Combined modality treatment } \\
(N=17)\end{array}$ & $\begin{array}{l}\text { Radiation therapy alone } \\
(N=63)\end{array}$ & $\begin{array}{l}\text { Systemic treatment } \\
(N=19)\end{array}$ \\
\hline \multirow[t]{2}{*}{ OS since diagnosis } & 5-year $(95 \% \mathrm{Cl})$ & $98.1 \%(0.945-1.000)$ & $100.0 \%(1.000-1.000)$ & $98.4 \%(0.952-1.000)$ & $84.2 \%(0.693-1.000)$ \\
\hline & $\mathrm{HR}^{\mathrm{a}}(95 \% \mathrm{Cl})$ & $\begin{array}{l}1.70(0.38-7.71) \\
p=0.49\end{array}$ & $\mathrm{NA}, p=1.00$ & Reference & $\begin{array}{l}4.85(1.05-22.36) \\
p=0.04\end{array}$ \\
\hline \multirow[t]{2}{*}{ PFS since treatment } & 5-year $(95 \% \mathrm{Cl})$ & \multirow[t]{2}{*}{-} & $80.0 \%(0.621-1.000)$ & $54.9 \%(0.438-0.688)$ & $44.7 \%(0.268-0.748)$ \\
\hline & $\mathrm{HR}^{\mathrm{a}}(95 \% \mathrm{Cl})$ & & $0.39(0.14-1.12), p=0.08$ & Reference & $\begin{array}{l}1.50(0.74-3.06) \\
p=0.26\end{array}$ \\
\hline
\end{tabular}

${ }^{a}$ Hazard ratio and corresponding $p$-value was adjusted for the Follicular Lymphoma International Prognostic Index (FLIPI). OS overall survival, HR hazard ratio, Inf infinity, PFS progression-free survival.

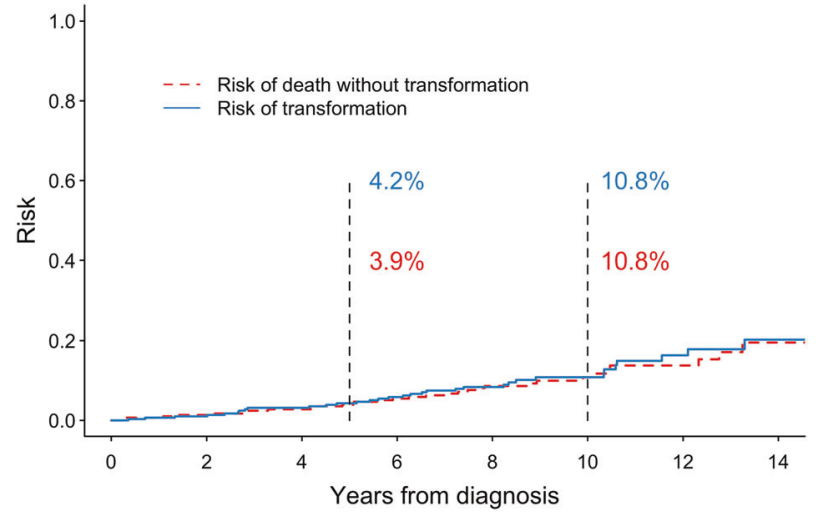

Fig. 5 Competing risk analysis of death and transformation. Competing risk analysis to demonstrate the rate of death without histological transformation and the rate of biopsy-proven histological transformation after diagnosis.

\section{DISCUSSION}

This study provides important information on early-stage $\mathrm{FL}$ and illustrates the survival patterns after management with different approaches, with extended follow-up in the modern era. Patients with deferred treatment underwent prolonged observation with a median duration of 5.62 years, and $43 \%$ of patients never required therapy by the time of data collection in 2017. The initial observation did not adversely affect survival compared with the immediately treated population. Similarly, the completeness of staging (presence or absence of PET and bone marrow examination) did not affect the lack of effect of initial observation on OS. After a median follow-up of 8.4 years, the 10 -year OS rate was $87 \%$ for all eligible patients. In patients with complete staging using PET and bone marrow biopsy, the 10 -year OS rate was $92 \%$. Patients treated with RT alone or CMT demonstrated prolonged survival, with 5 -year OS rates of $98.0 \%$ and $100.0 \%$, respectively. Progression free survival at 5 -years for all stage I-II patients was $78.9 \%, 62.3 \%$, and $45.7 \%$ in patients treated with CMT, RT alone, and systemic therapy, respectively but not statistically significant in completely staged patients.

PET has become the new paradigm in FL, and it provides essential information for staging, response assessment, and prognostication [12-16]. PET better identifies both nodal involvement and extra-nodal disease and upstages $24-62 \%$ of early-stage FL according to previous studies [13, 14]. Furthermore, PET reveals metabolic heterogeneity, which highlights the niche of suspected transformation to a disease of high aggressiveness. In combination with targeted biopsy, PET potentially influences the choice of initial therapy, the treatment outcome, and exclusion of these patients from accrual into prospective clinical trials or retrospective database analyses. This study confirmed the importance of PET imaging for localized FL. For the $52 \%$ of patients who were completely staged with PET and bone marrow biopsy, OS was significantly better than for provisionally staged patients (HR $0.45,95 \% \mathrm{Cl}$ 0.22-0.92, log-rank $p=0.02$ ).

Among patients with advanced-stage $\mathrm{FL}$, treatment in selected patients may be deferred without implication on survival [17]. The role of initial observation in patients with early-stage $\mathrm{FL}$, however, is less evident. In a previous retrospective study of 43 patients with observed stage I-II FL staged in the pre-PET era, the estimated 10 -year OS was $85 \%$ [4]. The choice of initial observation is desirable when significant co-morbidity is expected from immediate treatment. Our study confirms that initial observation is an appropriate option for early-stage FL patients and is not associated with inferior outcomes. 
Major guidelines have recommended RT for early-stage $\mathrm{FL}$, given the radiation-sensitive nature of FL [18-20]. Historically, the 5 -year OS rate of early-stage FL was reported to be $82-85 \%$ prior to routine radiographic staging with PET. In comparison, the 5-year OS rate for early-stage FL was $93-96 \%$ for patients staged in the PET era [5-8]. The survival after RT alone from the current study is again excellent, with $62.3 \%$ of patients free from treatment failure 5 years after treatment. Thus, our analysis advocates the incorporation of RT when treatment is indicated. Lymphoma progression generally occurred outside of the RT field, and attempts have been taken to increase long-term disease control for localized FL. Two randomized trials have been conducted to investigate the benefit of adding systemic therapy to RT in early-stage FL. The British National Lymphoma Investigation randomized trial found the addition of adjuvant oral chlorambucil to RT conferred no survival advantage [9]. In a phase III trial developed by the Trans-Tasman Radiation Oncology Group (TROG 99.03), patients with limited-stage FL were randomized to receive either involved-field radiotherapy (IFRT) or IFRT plus six cycles of cyclophosphamide, vincristine, and prednisolone (CVP) with or without rituximab. The addition of systemic chemotherapy was associated with better PFS (HR 0.57; $95 \% \mathrm{Cl} 0.34$ to $0.95 ; p=0.033$ ), although this was not reflected in an OS benefit. Enrollment onto TROG 99.03 was over a decade and while the proportion of PET imaging was relatively high, only $50 \%$ of patients had PET staging, and rituximab as a component of systemic therapy was only mandatory in a later amendment confounding its modern day applicability [10]. In a recently published analysis from the Australian Lymphoma Alliance, 365 stage I-II FL patients staged with PET demonstrated similar PFS in patients treated with RT alone and systemic therapy without maintenance rituximab (HR 1.32; $p=0.96$ ). The addition of maintenance rituximab to radiation therapy improved PFS (HR $0.24 ; P=0.017$ ) [5]. In the current study, patients treated with CMT demonstrated a similar OS to patients treated with radiation therapy alone. However, CMT was associated with a statistically better PFS in all eligible patients (HR $0.36 ; p=0.03$ ), although may not be powered to reveal difference in completely staged patients (HR 0.39; $p=0.08$ ). In a recent study where early-stage FL patients were evaluated for minimal residual disease (MRD) after involvedfield radiotherapy (IFRT), IFRT only induced MRD negativity in $40 \%$ of cases, suggesting that radiation therapy alone is insufficient to eradicate FL. Patients with persistent or relapsed MRD received ofatumumab, and future analysis with longer follow-up will better illuminate the role of biomarker-driven approaches in selecting patients who may benefit from combined modality therapy [21].

High-grade transformation to diffuse large B cell lymphoma is an integral part of FL's natural history and is associated with poor prognosis. Efforts have been made to identify FL patients at high risk for transformation before treatment commencement, including targeted biopsy guided by PET imaging. In our analysis, transformation events were confirmed by centrally reviewed pathological samples rather than solely by clinical criteria, and the competing risk analysis was performed to delineate the transformation patterns in early-stage FL. It revealed that the histological transformation rate steadily increases after diagnosis, with $4.2 \%$ of patients experiencing transformation at five years and $10.8 \%$ at one decade. Initial observation did not result in a higher rate of transformation compared with immediate treatment. Despite the perception of RT and CMT as possibly definitive therapies for FL, long-term patient surveillance may be required to monitor disease relapse and transformation.

Our study has the inherent weakness of a retrospective analysis. Unlike randomized trials, treatment approach to patients with early-stage $\mathrm{FL}$ included in a retrospective database was selected based on various factors presented at diagnosis, including stage, risk category, lesion location, co-morbidities and patients and physicians preferences. The baseline demographics differ across patients managed with different approaches in the current study. Comparing the outcome of patients having received systemic therapy with other groups is hampered by the small size of this group and the presence of patients with high-risk features. Future randomized trials are needed to compare outcomes after different treatment modalities.

In summary, early-stage FL has excellent overall survival after extended follow-up in the modern era. Selection for initial observation is independent of patients' age and does not adversely affect survival. Patients who received systemic regimen alone were identified to have high-risk baseline features, and overall survival after treatment was inferior to RT alone or CMT. These findings must be interpreted with caution, given the retrospective nature of the study and potential unmeasured confounding biases. Nevertheless, our data demonstrate that RT alone and CMT yielded excellent survival, and that CMT was associated with a better PFS. The definitive conclusion regarding the role of systemic regimen, especially the role of rituximab maintenance, needs to be addressed in future randomized trials incorporating modern imaging modality. Finally, the risk of transformation and disease relapse with early-stage FL increases over time; therefore, continued surveillance of early-stage FL patients is recommended.

\section{REFERENCES}

1. Morton LM, Wang SS, Devesa SS, Hartge P, Weisenburger DD, Linet MS. Lymphoma incidence patterns by WHO subtype in the United States, 1992-2001. Blood 2006;107:265-76.

2. Solal-Celigny $P$, Roy $P$, Colombat $P$, White J, Armitage JO, Arranz-Saez R, et al. Follicular lymphoma international prognostic index. Blood 2004;104:1258-65.

3. Soubeyran P, Eghbali H, Trojani M, Bonichon F, Richaud P, Hoerni B. Is there any place for a wait-and-see policy in stage 10 follicular lymphoma? A study of 43 consecutive patients in a single center. Ann Oncol. 1996;7:713-8.

4. Advani R, Rosenberg SA, Horning SJ. Stage I and II follicular non-Hodgkin's lymphoma: long-term follow-up of no initial therapy. J Clin Oncol. 2004;22:1454-9.

5. Tobin JWD, Rule G, Colvin K, Calvente L, Hodgson D, Bell S, et al. Outcomes of stage $1 /$ II follicular lymphoma in the PET era: an international study from the Australian Lymphoma Alliance. Blood Adv. 2019;3:2804-11.

6. Brady JL, Binkley MS, Hajj C, Chelius M, Chau K, Balogh A, et al. Definitive radiotherapy for localized follicular lymphoma staged by (18)F-FDG PET-CT: a collaborative study by ILROG. Blood 2019;133:237-45.

7. Mac Manus MP, Hoppe RT. Is radiotherapy curative for stage I and II low-grade follicular lymphoma? Results of a long-term follow-up study of patients treated at Stanford University. J Clin Oncol. 1996;14:1282-90.

8. Campbell BA, Voss N, Woods R, Gascoyne RD, Morris J, Pickles T, et al. Long-term outcomes for patients with limited stage follicular lymphoma: involved regional radiotherapy versus involved node radiotherapy. Cancer 2010;116:3797-806.

9. Kelsey SM, Newland AC, Hudson GV, Jelliffe AM. A British National Lymphoma Investigation randomised trial of single agent chlorambucil plus radiotherapy versus radiotherapy alone in low grade, localised non-Hodgkins lymphoma. Med Oncol. 1994;11:19-25.

10. MacManus M, Fisher R, Roos D, O'Brien P, Macann A, Davis $S$, et al. Randomized trial of systemic therapy after involved-field radiotherapy in patients with earlystage follicular lymphoma: TROG 99.03. J Clin Oncol. 2018;36:2918-25.

11. Gray RJ. A class of K-sample tests for comparing the cumulative incidence of a competing risk. Ann Stat. 1988;16:1141-54.

12. Le Dortz L, De Guibert S, Bayat S, Devillers A, Houot R, Rolland $Y$, et al. Diagnostic and prognostic impact of 18F-FDG PET/CT in follicular lymphoma. Eur J Nucl Med Mol Imaging. 2010;37:2307-14.

13. Luminari S, Biasoli I, Arcaini L, Versari A, Rusconi C, Merli F, et al. The use of FDGPET in the initial staging of 142 patients with follicular lymphoma: a retrospective study from the FOLL05 randomized trial of the Fondazione Italiana Linfomi. Ann Oncol. 2013;24:2108-12.

14. Metser U, Dudebout J, Baetz T, Hodgson DC, Langer DL, MacCrostie P, et al. [(18) F]-FDG PET/CT in the staging and management of indolent lymphoma: a prospective multicenter PET registry study. Cancer 2017;123:2860-6.

15. Trotman J, Barrington SF, Belada D, Meignan M, MacEwan R, Owen C, et al. Prognostic value of end-of-induction PET response after first-line immunochemotherapy for follicular lymphoma (GALLIUM): secondary analysis of a randomised, phase 3 trial. Lancet Oncol. 2018;19:1530-42. 
16. Batlevi CL, Sha F, Alperovich A, Ni A, Smith K, Ying Z, et al. Positron-emission tomography-based staging reduces the prognostic impact of early disease progression in patients with follicular lymphoma. Eur J Cancer. 2020;126:78-90.

17. Solal-Celigny $P$, Bellei $M$, Marcheselli $L$, Pesce EA, Pileri $S$, McLaughlin $P$, et al. Watchful waiting in low-tumor burden follicular lymphoma in the rituximab era: results of an F2-study database. J Clin Oncol. 2012;30:3848-53.

18. Dreyling M, Ghielmini $M$, Rule $S$, Salles $G$, Vitolo $U$, Ladetto $M$, et al. Newly diagnosed and relapsed follicular lymphoma: ESMO Clinical Practice Guidelines for diagnosis, treatment and follow-up. Ann Oncol. 2016;27:v83-v90.

19. McNamara C, Davies J, Dyer M, Hoskin P, Illidge $T$, Lyttelton $M$, et al. Guidelines on the investigation and management of follicular lymphoma. $\mathrm{Br} J$ Haematol. 2012;156:446-67.

20. NCCN guideline for the treatment of cancer: B-cell lymphomas https://www.nccn. org/professionals/physician_gls/default.aspx\#b-cell2019 [version 6.2019].

21. Pulsoni A, Tosti ME, Ferrero S, Luminari S, Liberati AM, Cenfra N, et al. Early stage follicular lymphoma: first results of the FIL "Miro" Study, a multicenter phase II trial combining local radiotherapy and MRD-driven immunotherapy [abstract]. Blood. 2019;134:124.

\section{AUTHOR CONTRIBUTIONS}

Conception and design: C.L.B., F.S., A.A., A.Y.; Collection and assembly of data: C.L.B., A.A., F.S.; Data analysis and interpretation: C.L.B., A.Y., G.S., F.S., V.E.S.; Manuscript writing: C.L.B., F.S., A.Y. contributed to writing the first draft of the manuscript. All authors provided a critical review of the manuscript's content and approved the final manuscript.

\section{FUNDING}

This research was funded in part by the Cancer Center Support Grant (Grant no. P30 CA008748) from the National Institutes of Health/National Cancer Institute to Memorial Sloan Kettering Cancer Center. Dr. Batlevi's work has been supported by an ASCO Young Investigator Award, an ASH Clinical Scholar Award, and the Lymphoma Research Foundation Clinical Research Mentoring Program.

\section{CONFLICT OF INTEREST}

Fushen Sha: None. Michelle Okwali: None. Anna Alperovich: None. Philip C. Caron: None. Lorenzo Falchi: Research funding from Roche, Genmab; Consultancy for Genmab. Audrey Hamilton: None. Paul A. Hamlin: research support from Portola Pharmaceuticals, Molecular Templates, Incyte, and J\&J Pharmaceuticals; and has a consultancy role with Portola Pharmaceuticals, Celgene, Karyopharm Therapeutics, and Juno Therapeutics. Steven M. Horwitz: research support from ADC Therapeutics, Aileron Therapeutics, Celgene, Forty Seven, Infinity Pharmaceuticals/Verastem Oncology, Kyowa Hakko Kirin, Millennium Pharmaceuticals/Takeda Oncology, Seattle Genetics, and Trillium; and has a consultancy role with ADC Therapeutics, Aileron, Corvus Pharmaceuticals, Forty Seven, Innate Pharma, Kyowa Hakko Kirin, Millennium/ Takeda, Mundipharma, Portola, and Seattle Genetics. Erel Joffe: Advisory board: AstraZeneca, Epizyme. Niloufer Khan: Research funding from Seattle Genetics. Anita Kumar: research support from AbbVie, Adaptive Biotechnologies, Celgene, Pharmacyclics, and Seattle Genetics; and has an advisory role with Celgene. Matthew J. Matasar: research support from Genentech, Roche, GlaxoSmithKline, Bayer, Pharmacyclics, Janssen, Rocket Medical, and Seattle Genetics; has received honoraria from Genentech, Roche, Bayer, Pharmacyclics, Janssen, Seattle Genetics, and GlaxoSmithKline; and has a consultancy role with Genentech, Bayer, Merck, Juno, Roche, Teva,
Rocket Medical, and Seattle Genetics. Alison J. Moskowitz: research support from Incyte, Seattle Genetics, Bristol-Myers Squibb, and Merck; and has a consultancy role with Kyowa Hakko Kirin Pharma, Miragen Therapeutics, Takeda Pharmaceuticals, ADC Therapeutics, Seattle Genetics, Cell Medica, Bristol-Myers Squibb, and Erytech Pharma. Ariela Noy: Research funding from Pharmacyclics, NIH, Raphael Pharma; consulting for Janssen, Pharmacyclics, Medscape, Targeted Oncology. Colette Owens: None. Lia M. Palomba: Honoraria from Merck, Celgene, Juno and Pharmacyclics. Ildefonso Rodriguez-Rivera: Stock/Ownership: Pfizer. David Straus: advisory role with Seattle Genetics. Gottfried von Keudell: has received research support from Pharmacyclics, Merck, Epizyme. Andrew D. Zelenetz: research support from MEI Pharma, MorphoSys, Sandoz, Celgene, Roche, and Gilead Sciences; has a consultancy role with Genentech/Roche, Gilead, Celgene, Janssen, Amgen, Novartis, and Adaptive Biotechnologies; and serves on the board of directors (Data Monitoring Committee Chair) for BeiGene. Joachim Yahalom: none. Ahmet Dogan: personal fees from Corvus Pharmaceuticals, Physicians' Education Resource, Seattle Genetics, Takeda, Roche, EUSAPharma, PeerView, and research support from Roche and Takeda. Heiko Schoder: none. Venkatraman E. Seshan: none. Gilles Salles: consultancy role with Abbvie, Bayer, Beigene, BMS/Celgene, Debiopharm, Epizyme, Genentech/Roche, Genmab, Incyte, Ipsen, Janssen, Kite/Gilead, Loxo, Milteniy, Morphosys, Novartis, Rapt, Regeneron, Takeda, Velosbio. Anas Younes: employment at AstraZeneca. Connie L. Batlevi: research support from Janssen, Novartis, Epizyme, Autolus, Roche, and Bayer; has received honoraria from Dava Oncology; and has a consultancy role with Skipta, Kite Pharma, MorphoSys, Bristol-Myers Squibb, Karyopharm Therapeutics, Genentech, and TG Therapeutics.

\section{ADDITIONAL INFORMATION}

Supplementary information The online version contains supplementary material available at https://doi.org/10.1038/s41408-022-00620-w.

Correspondence and requests for materials should be addressed to Connie L. Batlevi.

Reprints and permission information is available at http://www.nature.com/ reprints

Publisher's note Springer Nature remains neutral with regard to jurisdictional claims in published maps and institutional affiliations.

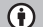

Open Access This article is licensed under a Creative Commons Attribution 4.0 International License, which permits use, sharing, adaptation, distribution and reproduction in any medium or format, as long as you give appropriate credit to the original author(s) and the source, provide a link to the Creative Commons license, and indicate if changes were made. The images or other third party material in this article are included in the article's Creative Commons license, unless indicated otherwise in a credit line to the material. If material is not included in the article's Creative Commons license and your intended use is not permitted by statutory regulation or exceeds the permitted use, you will need to obtain permission directly from the copyright holder. To view a copy of this license, visit http://creativecommons. org/licenses/by/4.0/.

(c) The Author(s) 2022 